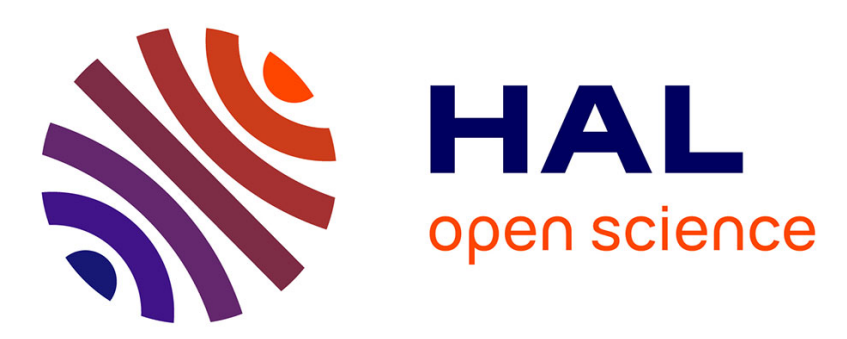

\title{
Fronts between periodic patterns for bistable recursions on lattices
}

\author{
Ricardo Coutinho, Bastien Fernandez
}

\section{To cite this version:}

Ricardo Coutinho, Bastien Fernandez. Fronts between periodic patterns for bistable recursions on lattices. Journal of Dynamics and Differential Equations, 2013, 25, pp.17-31. 10.1007/s10884-0129285-y . hal-00660246

\section{HAL Id: hal-00660246 https://hal.science/hal-00660246}

Submitted on 16 Jan 2012

HAL is a multi-disciplinary open access archive for the deposit and dissemination of scientific research documents, whether they are published or not. The documents may come from teaching and research institutions in France or abroad, or from public or private research centers.
L'archive ouverte pluridisciplinaire HAL, est destinée au dépôt et à la diffusion de documents scientifiques de niveau recherche, publiés ou non, émanant des établissements d'enseignement et de recherche français ou étrangers, des laboratoires publics ou privés. 


\title{
Fronts between periodic patterns for bistable recursions on lattices
}

\author{
Ricardo Coutinho* $^{*,}$ and Bastien Fernandez ${ }^{\dagger}$ \\ * Grupo de Física Matemática \\ Universidade de Lisboa \\ 1649-003 Lisboa, Portugal \\ $\S$ Departamento de Matemática \\ Instituto Superior Técnico \\ Universidade Técnica de Lisboa \\ 1049-001 Lisboa, Portugal \\ $\dagger$ Centre de Physique Théorique, UMR 7332 \\ CNRS - Aix-Marseille Université \\ Campus de Luminy \\ 13288 Marseille CEDEX 9, France
}

\begin{abstract}
Bistable space-time discrete systems commonly possess a large variety of stable stationary solutions with periodic profile. In this context, it is natural to ask about the fate of trajectories composed of interfaces between steady configurations with periodic pattern and in particular, to study their propagation as traveling fronts. Here, we investigate such fronts in piecewise affine bistable recursions on the one-dimensional lattice. By introducing a definition inspired by symbolic dynamics, we prove the existence of front solutions and uniqueness of their velocity, upon the existence of their ground patterns. Moreover, the velocity dependence on parameters and the co-existence of several fronts with distinct ground patterns are also described. Finally, robustness of the results to small $C^{1}$-perturbations of the piecewise affine map is argued by mean continuation arguments.
\end{abstract}

Keywords: Periodic fronts, Bistable recursions, Space-time discrete systems, Symbolic dynamics. January 16, 2012.

\section{Introduction}

Invasion phenomena are ubiquitous in spatially extended systems. The introduction of a stable germ inside an unstable (or less stable) phase usually results in the propagation of interfaces that progressively take the form of traveling fronts with monotonic profile. This phenomenon typically occurs in, for instance, alloy solidification, redox chemical reactions or the spreading of infectious diseases.

Since the pioneering work of Kolmogorov, Petrovsky and Piskunov in 1937, front propagation has been mathematically investigated in a large variety of systems. Examples range from parabolic PDEs (see e.g. $[4,11,23]$ and references therein), to integro-differential equations, e.g. [6, 9], to lattice models of coupled ODEs $[5,26,29,34]$ and to discrete-time models of recursions $[18,28,30]$. A specific feature of spatially discrete/lattice models is the so-called pinning effect: (bistable) front propagation fails when the relative asymmetry of the two stable phases is insufficiently large. This property contrasts with PDEs where propagation occurs as soon as the bistable nonlinearity becomes asymmetric.

Beyond their de facto relevance for numerical simulations, discrete-time systems can be regarded as basic versions of models with delays or with periodic updating [22, 27]. Moreover, Poincaré sections and time-1 maps techniques certify that, at least formally, the dynamics of recursions contains all trajectories of PDE's and integro-differential equations. On the other hand, lattice models naturally arise when representing systems composed of markedly discrete units such as in solid-state physics or in traffic flows, but also when modeling involves some local averaging in space. 
Discreteness of space in lattice systems is responsible for robust spatial chaos, namely the existence of cohorts of stationary solutions with distinct profiles that are stable with respect to parameter changes. For instance, a discrete reaction-diffusion system with bistable nonlinearity has, upon choice of parameters, stable stationary solutions with periodic profiles, for (almost) every integer period [1, 16, 20], in addition to the two stable translation invariant solutions inherited from the local nonlinear reaction. (Spatial chaos also exists in PDEs with symmetric nonlinear reactions $[13,21]$ but it is unstable to arbitrary small asymmetric perturbations.) This phenomenon is usually analyzed by means of horseshoe construction for the "spatial" dynamical system associated with stationary solutions $[3,10]$.

By analogy with standard fronts, the existence of robust stationary solutions with periodic profile in lattice models suggests to investigate fronts that propagate between two such ground patterns. What about the invasion of a spatially periodic phase by another one, via an interface that moves regularly along the lattice, like a traveling wave?

Ground pattern periodicity suggests to call up periodic/pulsating fronts as they have been proved to occur in periodic (continuous) media [7, 24, 32]. Indeed, the concept of periodic fronts naturally emerges in discrete systems when the lattice step is regarded as an intrinsic spatial period. Actually, monostable periodic fronts have been studied for some spatially periodic recursions on lattices [25, 31].

In order to accommodate the potentially large variety of ground patterns that may coexist in spacetime discrete bistable systems and in particular, the occurrence of periodic profiles with period equal to an arbitrary multiple of the lattice step, we consider here a more specific description of periodic fronts. Our definition relies on the symbolic dynamics analysis of the recursion and explicitly incorporates ground patterns as varying parameters via their codes, see equation (11) below. This approach is particularly suited to describe the front velocity dependence on system parameters (once the pair of ground patterns has been fixed). It is also convenient to study the co-existence of several fronts when spatial chaos takes place.

More specifically, we study the dynamics of fronts between periodic patterns in parameter families of bistable piecewise affine recursions on one-dimensional lattices. We prove the existence of such solutions (upon ground pattern existence, as it is the case for periodic fronts in continuous media), the uniqueness of their velocity, and their stability (for almost all parameter values in their existence domain). In other words, relatively to ground pattern existence domains in parameter space, these fronts are shown to have a similar phenomenology to standard fronts between constant stable phases. We also describe the velocity dependence on parameters and the co-existence with standard fronts and, possibly, other fronts between periodic patterns. Finally, we mention in the last section, those results that are not specific to piecewise affine systems and extend to more general smooth recursions.

\section{Bistable piecewise affine recursions on one-dimensional lattices and their symbolic dynamics}

Our framework is that of recursions (discrete-time dynamical system) acting on the space of configurations $\left\{u_{s}\right\}_{s \in \mathbb{Z}}$ (with components $u_{s}$ ) of the one-dimensional lattice $\mathbb{Z}$, endowed with supremum norm $\|\cdot\|$. Temporal orbits $\left\{u^{t}\right\}_{t \geqslant 0}$ (or $\left\{u^{t}\right\}_{t \in \mathbb{Z}}$ depending on context) are composed of lattice configurations $\left(u^{t}=\right.$ $\left\{u_{s}^{t}\right\}_{s \in \mathbb{Z}}$ for all $t$ ). We consider translation invariant recursions that are performed by the lattice convolution of the images of components by some bistable nonlinearity $f_{a, c}$, namely

$$
u_{s}^{t+1}=\sum_{n \in \mathbb{Z}} \ell_{n} f_{a, c}\left(u_{s-n}^{t}\right), \forall s \in \mathbb{Z}
$$

Here the coefficients $\ell_{n}$ are chosen non-negative and non-trivial (i.e. $0 \leqslant \ell_{n}<1$ ) and normalized (i.e. $\left.\sum_{n \in \mathbb{Z}} \ell_{n}=1\right)$. These assumptions ensure that the coupling operator $C$, defined as the following linear and bounded convolution operator acting on $\ell^{\infty}(\mathbb{Z})$

$$
(C u)_{s}=\sum_{n \in \mathbb{Z}} \ell_{n} u_{s-n}, \forall s \in \mathbb{Z}
$$

behaves as a diffusion operator (and is neither the identity nor a pure translation). The map $f_{a, c}$ is defined by [17] (see Figure 1) 


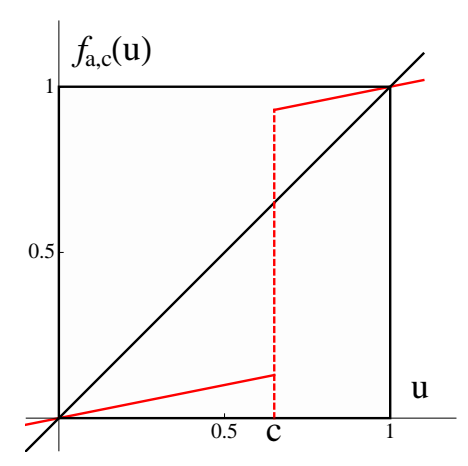

Figure 1: Graph of the bistable nonlinearity $f_{a, c}(a=0.2$ and $c=0.65)$.

$$
f_{a, c}(u)=a u+(1-a) H(u-c), \forall u \in \mathbb{R}
$$

where $H$ is the Heaviside function (i.e. $H(u)=0$ if $u<0$ and $H(u)=1$ if $u \geqslant 0$ ) and $0 \leqslant a<1$ and $0<c<1$. In particular 0 and 1 are stable fixed points of $f_{a, c}$ and the discontinuity $c$ plays the role of a repeller for this map.

Clearly, the assumptions on the $\ell_{n}$ and the expression of $f_{a, c}$ imply that for any initial configuration $u^{0} \in$ $\ell^{\infty}(\mathbb{Z})$, the subsequent orbit $\left\{u^{t}\right\}_{t \geqslant 0}$ is well-defined, that is to say the dynamics (1) is well-defined.

Example: Nearest neighbor symmetric coupling. A basic example of recursion (1) is given by $\ell_{n}=$ $(1-\epsilon) \delta_{n, 0}+\frac{\epsilon}{2}\left(\delta_{n,-1}+\delta_{n, 1}\right)$ with $0<\epsilon \leqslant 1$, viz.

$$
u_{s}^{t+1}=(1-\epsilon) f_{a, c}\left(u_{s}^{t}\right)+\frac{\epsilon}{2}\left(f_{a, c}\left(u_{s-1}^{t}\right)+f_{a, c}\left(u_{s+1}^{t}\right)\right), \forall s \in \mathbb{Z} .
$$

Such discrete time systems (with arbitrary real map $f_{a, c}$ ) are well-known under the appellation of Coupled Map Lattices [8] and have been extensively studied in the nonlinear science and dynamical systems communities.

The assumptions on $\left\{\ell_{n}\right\}_{n \in \mathbb{Z}}$ and the bistability of $f_{a, c}$ also imply that both constant configurations $u_{s}=0$ for all $s$ and $u_{s}=1$ for all $s$, which we respectively abbreviate as $0^{\infty}$ and $1^{\infty}$, are (asymptotically) stable fixed points of the dynamics. In fact, these translation invariant stationary solutions constitute the ground patterns of standard fronts. Standard fronts in piecewise affine bistable recursions have been thoroughly investigated in [15] in the case of nearest neighbor symmetric coupling, and in [17] in the case of arbitrary coupling sequence $\left\{\ell_{n}\right\}_{n \in \mathbb{Z}}$.

An important technique for the study of piecewise affine systems is symbolic dynamics. Symbolic dynamics relies on a coding of trajectories. In our context, this consists in associating, via the individual map partition, a symbolic (space-time) sequence $\left\{\theta_{s}^{t}\right\}_{s \in \mathbb{Z}, t \geqslant 0}\left(\right.$ or $\left.\left\{\theta_{s}^{t}\right\}_{(s, t) \in \mathbb{Z}^{2}}\right)$ with $\theta_{s}^{t} \in\{0,1\}$ to every orbit of the recursion (1), viz.

$$
\theta_{s}^{t}=H\left(u_{s}^{t}-c\right), \forall s \in \mathbb{Z}, t \geqslant 0 \text { (or } t \in \mathbb{Z} \text { ). }
$$

For piecewise affine systems, introducing the coding allows one to formally solve the induction (1) for bounded bi-infinite orbits $\left\{u^{t}\right\}_{t \in \mathbb{Z}}$ and to obtain a self-consistency equation for their code. This equation is called the admissibility equation (see (5) below).

Beside their convenience for the symbolic dynamics analysis, bounded bi-infinite orbits have interest in their own, at least when the coupling operator $C$ is invertible with bounded inverse. Indeed, the recursion (1) is injective on its image in this case, because the nonlinearity $f_{a, c}$ is itself one-to-one. Using this property, it can be shown that the set of all configurations of bounded bi-infinite orbits coincides with the (standard) attractor of the recursion (see [19] for a proof in finite dimension; a proof that applies mutatis mutandis to the dynamical system here). For instance, the nearest neighbor symmetric coupling in equation (2) is invertible with bounded inverse for every $\epsilon<1 / 2$. In the general case, this condition holds provided that $C$ is sufficiently close to the identity. 
In order to write the admissibility equation, we need to consider the entries of the powers $C^{k}$ of the coupling operator, namely the coefficients $\left\{\ell_{n, k}\right\}_{n \in \mathbb{Z}}$ defined by

$$
\left(C^{k} u\right)_{s}=\sum_{n \in \mathbb{Z}} \ell_{n, k} u_{s-n}, \forall s \in \mathbb{Z}, k \geqslant 1 .
$$

In particular, we have $\ell_{n, 1}=\ell_{n}$ for all $n$ and, since $C$ is a convolution operator, the entries for $k \geqslant 2$ are defined via the following induction [2]

$$
\ell_{n, k+1}=\sum_{m \in \mathbb{Z}} \ell_{m} \ell_{n-m, k}, \forall n \in \mathbb{Z}, k \geqslant 1 .
$$

This relation easily implies that the entries must be all non-negative and non-trivial (i.e. $\left.0 \leqslant \ell_{n, k}<1\right)$ and normalized (i.e. $\sum_{n \in \mathbb{Z}} \ell_{n, k}=1$ for all $k$ ).

Anticipating the result of solving the induction (1), the component function $\phi$ of the (putative) bounded orbit associated with the bi-infinite symbolic sequence $\vartheta=\left\{\theta_{s}^{t}\right\}_{(s, t) \in \mathbb{Z}^{2}} \in\{0,1\}^{2}$ is given by

$$
\phi(\vartheta)=\frac{1-a}{a} \sum_{k=1}^{+\infty} a^{k} \sum_{n \in \mathbb{Z}} \ell_{n, k} \theta_{-n}^{-k}
$$

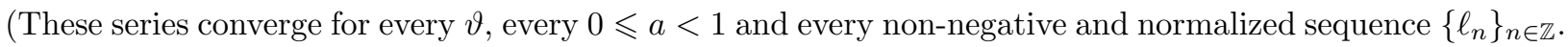
Moreover, $\phi$ only depends on $\left\{\theta_{s}^{t}\right\}_{s \in \mathbb{Z}, t \leqslant 0}$ but the dependence on the whole sequence will become clear in the Lemma below.) Let $R_{\mathrm{sp}}$ and $R_{\mathrm{ti}}$ respectively denote the space and time translations acting on symbolic sequences, i.e.

$$
\left(R_{\mathrm{sp}} \vartheta\right)_{s}^{t}=\theta_{s+1}^{t} \quad \text { and } \quad\left(R_{\mathrm{ti}} \vartheta\right)_{s}^{t}=\theta_{s}^{t+1}, \forall(s, t) \in \mathbb{Z}^{2}
$$

Lemma 1 A bounded bi-infinite sequence $\left\{u^{t}\right\}_{t \in \mathbb{Z}}$ of configurations in $\ell^{\infty}(\mathbb{Z})$ (i.e. such that $\sup _{t \in \mathbb{Z}}\left\|u^{t}\right\|<+\infty$ ) satisfies the recursion (1) for all $t \in \mathbb{Z}$ iff its components write

$$
u_{s}^{t}=\phi \circ R_{\mathrm{ti}}^{t} \circ R_{\mathrm{sp}}^{s}(\vartheta), \forall(s, t) \in \mathbb{Z}^{2}
$$

where $\vartheta$ is the associated code. Independently, given a symbolic sequence $\vartheta$, the sequence $\left\{\phi \circ R_{\mathrm{ti}}^{t} \circ R_{\mathrm{sp}}^{s}(\vartheta)\right\}(s, t) \in \mathbb{Z}^{2}$ satisfies the recursion (1) for all $t \in \mathbb{Z}$ iff $\vartheta$ solves the equation

$$
\theta_{s}^{t}=H\left(\phi \circ R_{\mathrm{ti}}^{t} \circ R_{\mathrm{sp}}^{s}(\vartheta)-c\right), \forall(s, t) \in \mathbb{Z}^{2} .
$$

As we shall see below, the admissibility equation (5) appears to be convenient in the analysis of bounded orbits associated with fronts.

Proof of the Lemma. We only show that if $u^{t}$ belongs to a bounded orbit, then it writes $\left\{\phi \circ R_{\mathrm{ti}}^{t} \circ R_{\mathrm{sp}}^{s}(\vartheta)\right\}_{s \in \mathbb{Z}}$. The proofs of other properties are direct and left to the reader.

By introducing the notation $\theta^{t}=\left\{\theta_{s}^{t}\right\}_{s \in \mathbb{Z}}$ for configurations of symbols, the recursion (1) can be written in an operator form as follows

$$
u^{t}=a C u^{t-1}+(1-a) C \theta^{t-1} .
$$

Iterating backward yields the following expression

$$
u^{t}=a^{n} C u^{t-n}+\frac{1-a}{a} \sum_{k=1}^{n} a^{k} C^{k} \theta^{t-k}, \forall n \geqslant 1 .
$$

We have $\|C\|=1$. Therefore, the assumption $\sup _{t \in \mathbb{Z}}\left\|u^{t}\right\|=M<+\infty$ for the orbit under consideration implies

$$
a^{n}\left\|C u^{t-n}\right\| \leqslant a^{n}\left\|u^{t-n}\right\| \leqslant a^{n} M, \forall n \geqslant 1 .
$$

By taking the limit $n \rightarrow+\infty$ in the expression of $u^{t}$ above, we obtain the following formula for its components

$$
u_{s}^{t}=\frac{1-a}{a} \sum_{k=1}^{+\infty} a^{k}\left(C^{k} \theta^{t-k}\right)_{s}=\frac{1-a}{a} \sum_{k=1}^{+\infty} a^{k} \sum_{n \in \mathbb{Z}} \ell_{n, k} \theta_{s-n}^{t-k}, \forall(s, t) \in \mathbb{Z}^{2}
$$

which is the desired expression. 


\section{Existence conditions for stationary configurations}

As mentioned above, the existence of fronts depends upon the existence of their ground patterns. Ground patterns correspond to stationary orbits (fixed points) of the recursion. These solutions are special cases of bounded bi-infinite sequences $\left\{u^{t}\right\}_{t \in \mathbb{Z}}$ for which the code is independent of $t\left(\theta^{t}=\theta\right.$ for all $\left.t\right)$ and thus only depend on spatial coordinates. Keeping the same notation $\phi$, the component function associated with a spatial code $\theta=\left\{\theta_{s}\right\}_{s \in \mathbb{Z}}$ naturally writes

$$
\phi(\theta)=\sum_{n \in \mathbb{Z}} l_{n} \theta_{-n}
$$

where $l_{n}=\frac{1-a}{a} \sum_{k=1}^{+\infty} a^{k} \ell_{n, k}$. Alternatively these coefficients $l_{n}$ can be viewed as the entries of the operator $(1-a) C(\operatorname{Id}-a C)^{-1}$ (see relation (6) above) which is itself a convolution operator [2], i.e. for every $u \in \ell^{\infty}(\mathbb{Z})$, we have

$$
\left((1-a) C(\operatorname{Id}-a C)^{-1} u\right)_{s}=\sum_{n \in \mathbb{Z}} l_{n} u_{s-n}, \forall s \in \mathbb{Z} .
$$

According to Lemma 1, the components of the stationary orbit associated with $\vartheta$ write (where we also keep the notation $R_{\mathrm{sp}}$ for the translation acting on spatial codes)

$$
u_{s}=\phi \circ R_{\mathrm{sp}}^{s}(\theta), \forall s \in \mathbb{Z}
$$

and the admissibility equation for $\theta$ is

$$
\theta_{s}=H\left(\phi \circ R_{\mathrm{sp}}^{s}(\theta)-c\right), \forall s \in \mathbb{Z} .
$$

It is convenient to re-write this condition as follows

$$
M(\theta) \lesssim c \leqslant m(\theta) \quad \text { where } \quad M(\theta)=\sup _{s: \theta_{s}=0} \phi \circ R_{\mathrm{sp}}^{s}(\theta) \quad \text { and } \quad m(\theta)=\inf _{s: \theta_{s}=1} \phi \circ R_{\mathrm{sp}}^{s}(\theta)
$$

and where the symbol $\lesssim$ means $<$ if $M$ is a maximum and it means $\leqslant$ if this supremum is not attained. Therefore, whenever $M(\theta)<m(\theta)$, there exists an interval of the parameter $c$ for which the fixed point associated with $\theta$ exists.

The property $M(\theta)<m(\theta)$ is not rare and can in fact be satisfied on open sets of parameters $a$ and $\left\{\ell_{n}\right\}_{n \in \mathbb{Z}}$. In particular, if the original coupling operator $C$ is sufficiently close to the identity (see below) such that $l_{0}>1 / 2$, then we have

$$
\sup _{\theta \in\{0,1\}^{\mathbb{Z}}} M(\theta)=1-l_{0}<l_{0}=\inf _{\theta \in\{0,1\}^{\mathbb{Z}}} m(\theta)
$$

and thus for $c \in\left(1-l_{0}, l_{0}\right]$, all spatial codes are admissible (and there is a spatial chaos of maximal diversity in this case).

To show that $l_{0}>1 / 2$ when $C$ is close to the identity, notice that the induction relation (3), together with non-negativity of the coefficients, implies the inequality $\ell_{0, k+1} \geqslant \ell_{0} \ell_{0, k}$, which in turn gives $\ell_{0, k} \geqslant \ell_{0}^{k}$ for all $k \geqslant 1$. The definition of $l_{n}$ at the beginning of this section then yields

$$
l_{0}=\frac{1-a}{a} \sum_{k=1}^{+\infty} a^{k} \ell_{0, k} \geqslant \frac{1-a}{a} \sum_{k=1}^{+\infty} a^{k} \ell_{0}^{k}=\frac{1-a}{1-a \ell_{0}} \ell_{0}
$$

from which it follows that $l_{0}>1 / 2$ when $1-\ell_{0}=\frac{\|\mathrm{Id}-C\|}{2}<\frac{1-a}{2-a}$ as desired.

\section{Simplest front between periodic patterns}

In this piecewise affine setting, a standard front with velocity $v$ is the bounded orbit associated with a purely interfacial code, i.e. such that there exists $x_{0} \in \mathbb{R}$ such that $\vartheta$ is given by

$$
\theta_{s}^{t}=H\left(s-v t+x_{0}\right), \forall(s, t) \in \mathbb{Z}^{2} .
$$


The quantity $x_{0}$ is only a phase parameter that has no influence on the existence of fronts. Furthermore, the front itself writes as a pure traveling wave (i.e. $\left.u_{s}^{t}=\phi\left(s-v t+x_{0}\right)\right)$. In a space-time discrete system, this expression is more general than requiring that the orbit be space-time periodic (i.e. $u_{s}^{t+q}=u_{s+p}^{t}$ for some integer pair $(p, q))$, because it includes irrational velocities, while space-time periodicity does not.

For a standard front, the ground patterns are the constant configurations $0^{\infty}$ and $1^{\infty}$ (which respectively correspond to the spatial codes $\theta_{s}=0$ for all $s$ and $\theta_{s}=1$ for all $s$ ). The simplest extension of a standard front is a traveling wave that represents the invasion of the constant phase $0^{\infty}$ into the ground pattern with spatial period 2. This pattern is the stationary configuration associated with the spatial code $\bar{\theta}$ defined by (and which we abbreviate as $(10)^{\infty}$ )

$$
\bar{\theta}_{s}=\frac{1+(-1)^{s}}{2}, \forall s \in \mathbb{Z} .
$$

The front is the bounded orbit associated with the code

$$
\theta_{s}^{t}=H\left(s-v t+x_{0}\right) \frac{1+(-1)^{s}}{2}, \forall(s, t) \in \mathbb{Z}^{2}
$$

A snapshot of this front in the case of the nearest neighbor symmetric coupling recursion, together with a standard front profile, are given on Figure 2 (see also supplementary information [14] for computer animations of their time evolution). Notice that a similar solution has been observed in the modeling of neural fields $[12]$.
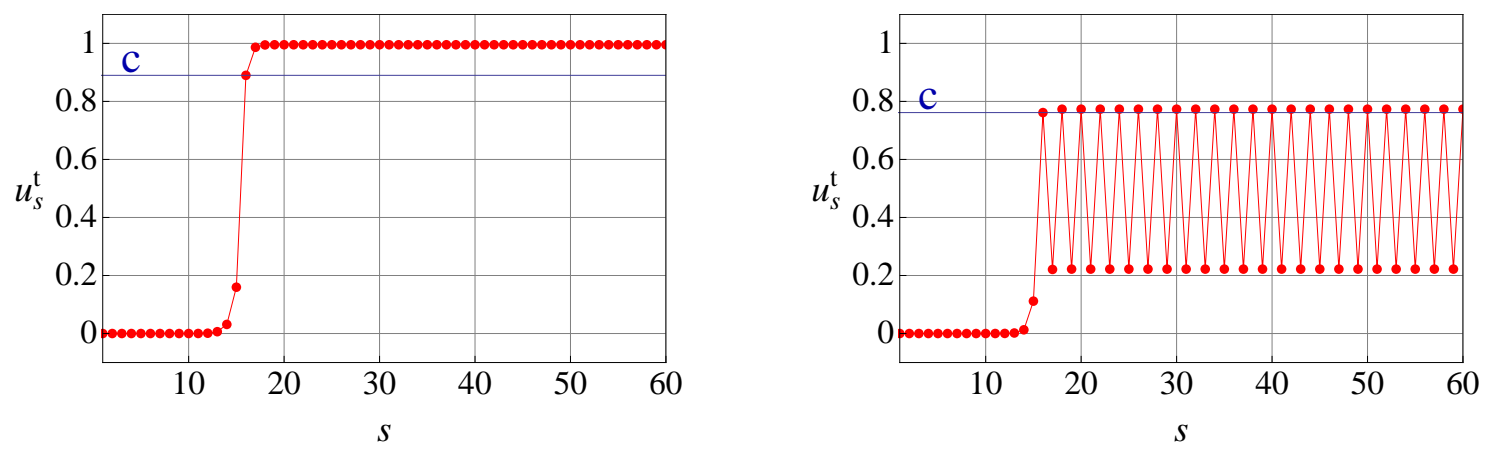

Figure 2: Standard front profile (left) and a snapshot (right) of the front (8) between $0^{\infty}$ and $(10)^{\infty}$ for the recursion (2). The front velocity is $v=4 / 23$ in both cases and the system parameters are $a=0.8, \epsilon=0.07$ and $c=0.890$ (left) $/ c=0.762$ (right).

The analysis of the admissibility equation for this code results in the following statement on front existence, uniqueness of velocity and velocity continuity with respect to parameters. As anticipated, the existence statement is relative to ground pattern existence.

Proposition 2 Assume that the parameter a and $\left\{\ell_{n}\right\}_{n \in \mathbb{Z}}$ are such that the inequality $M(\bar{\theta})<m(\bar{\theta})$ holds (necessary condition for existence of the stationary solution $\bar{\theta}=(10)^{\infty}$ ). Then the following assertions hold.

(i) There exists a countable and nowhere dense set $G$ such that the code (8) solves the admissibility equation (5) for some velocity $v \in \mathbb{R}$ iff $c \in[M(\bar{\theta}), m(\bar{\theta})] \backslash G$.

(ii) For every $c \in[M(\bar{\theta}), m(\bar{\theta})] \backslash G$, there exists a unique $v(c) \in \mathbb{R}$ for which (8) solves (5) (i.e. the corresponding velocity is unique).

(iii) The function $v(\cdot)$ can be extended to the whole interval $[M(\bar{\theta}), m(\bar{\theta})]$ as an increasing Devil's staircase. It also continuously depends on a and $\left\{\ell_{n}\right\}_{n \in \mathbb{Z}}$.

Notice that the inequality $M(\bar{\theta})<m(\bar{\theta})$ is equivalent to $\sum_{n \in \mathbb{Z}} l_{2 n+1}<\sum_{n \in \mathbb{Z}} l_{2 n}$. It certainly holds when the coupling operator $C$ sufficiently close to the identity. 
In addition, the proof of this statement shows that for $c \in G$, the recursion (1) has 'ghost' fronts, namely bounded bi-infinite front-like sequences in phase space that do not satisfy the relation (1) (but which would satisfy it if we had $H(0)=0$ instead of $H(0)=1)$. In particular, some ghost front component(s) must lie on the discontinuity $c$. Despite that they are not genuine orbits, ghost fronts are relevant objects in this discontinuous system because they attract open sets of initial conditions (see [15, 17] for more details on ghost (standard) fronts).

The proof also proffers an explicit expression for the front velocity, see (9) below. This expression is illustrated by Figure 3 which displays a color plot in the square $(\epsilon, c)$ for $a$ fixed, of front velocities for the nearest neighbor recursion (2). The picture clearly reveal the staircase structure of the function $c \mapsto v(c)$, with an $\epsilon$-dependent range. In addition, Figure 3 shows that different fronts may propagate with distinct velocities (e.g. fronts between $0^{\infty}$ and $(10)^{\infty}$ with non-vanishing velocity can only exist in the zero velocity region of standard fronts).
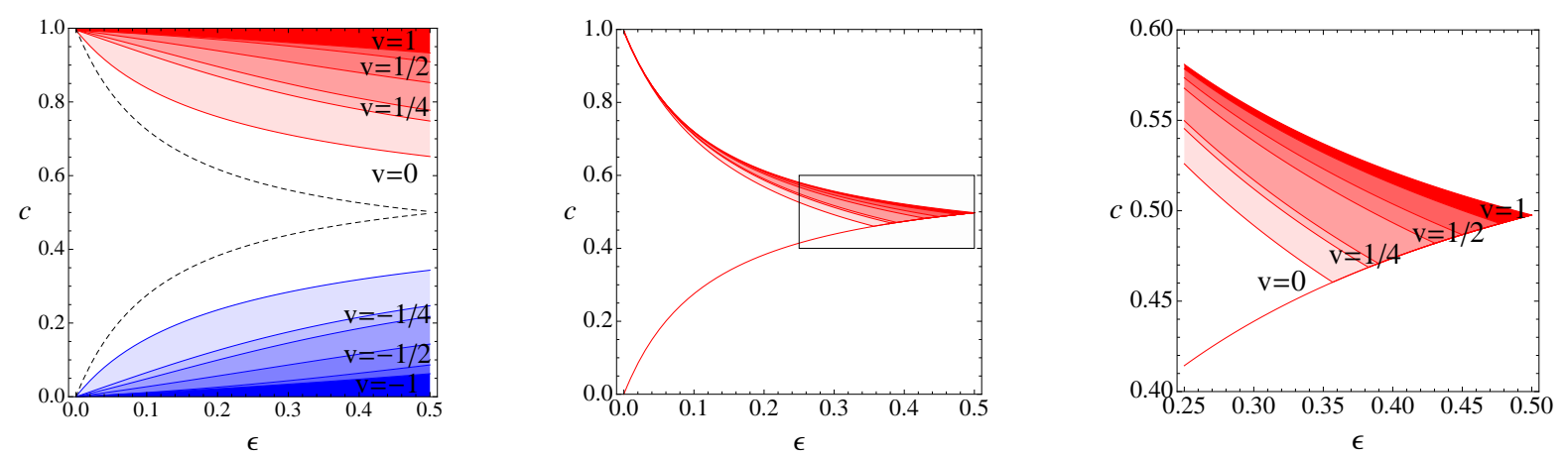

Figure 3: Existence domains in the $(\epsilon, c)$ plane for $a=0.8$, of standard fronts (left picture) and fronts (8) between $0^{\infty}$ and $(10)^{\infty}$ (middle and right pictures; the right picture is a magnification of the rectangle in the middle one) for the recursion (2). Colors code for velocities, from -1 (dark blue), to 0 (white) and to 1 (dark red). Some of the domains where the velocity has a given rational velocity are shown. (Between those domains, the velocity takes every intermediate value, in particular it can be an irrational number.) The middle and right pictures have been computed using the inequalities (9). The top (resp. bottom) curve represents the function $\epsilon \mapsto M(\bar{\theta})$ (resp. $\epsilon \mapsto m(\bar{\theta})$ ) - also shown as dashed curves in the left picture - beyond which no front (8) can exist. The left picture has been computed by using analytic expressions in [15], (see also proof of Theorem 3 below). Notice that standard fronts exist for every $c \in[0,1] \backslash G_{1, \emptyset}$ where $G_{1, \emptyset}$ is a countable and nowhere dense set.

Proof of the Proposition. The front code (8) can be expressed as

$$
\theta_{2 s}^{t}=H\left(2 s-v t+x_{0}\right) \quad \text { and } \quad \theta_{2 s+1}^{t}=0, \forall(s, t) \in \mathbb{Z}^{2}
$$

By inserting this expression into the component function (4), one easily obtains that the associated front components write

$$
u_{2 s+r}^{t}=\phi_{r}\left(2 s-v t+x_{0}, v\right), \forall r \in\{0,1\},(s, t) \in \mathbb{Z}^{2}
$$

where

$$
\phi_{r}(x, v)=\frac{1-a}{a} \sum_{k=1}^{+\infty} a^{k} \sum_{n \in \mathbb{Z}} \ell_{2 n+r, k} H(x-2 n+v k), \forall x, v \in \mathbb{R} .
$$

The coefficients $\ell_{n, k} \geqslant 0$ are non-negative; hence both functions $x \mapsto \phi_{r}(x, v)$ are right continuous and increasing.

The admissibility equation for the code (8) is equivalent to

$$
\begin{aligned}
& \sup _{s, t: 2 s-v t+x_{0}<0} \phi_{0}\left(2 s-v t+x_{0}, v\right) \lesssim c \leqslant \inf _{s, t: 2 s-v t+x_{0} \geqslant 0} \phi_{0}\left(2 s-v t+x_{0}, v\right) \\
& \text { and } \phi_{1}\left(2 s-v t+x_{0}, v\right)<c, \forall(s, t) \in \mathbb{Z}^{2} .
\end{aligned}
$$


Let $n_{\max }=\sup \left\{n: \ell_{n}>0\right\}$. A direct analysis shows that we have

$$
\phi_{1}(x, v)<\phi_{1}(+\infty, v), \forall x \in \mathbb{R} \text { if } v<n_{\max } \quad \text { and } \quad \phi_{1}\left(0, n_{\max }\right)=\phi_{1}\left(+\infty, n_{\max }\right) \text { if } n_{\max }<+\infty .
$$

In addition, for all $v \in \mathbb{R}$, we have $\phi_{1}(+\infty, v)=\frac{1-a}{a} \sum_{k=1}^{+\infty} a^{k} \sum_{n \in \mathbb{Z}} \ell_{2 n+1, k}=\sum_{n \in \mathbb{Z}} l_{2 n+1}=M(\bar{\theta})$. Hence the last admissibility condition is equivalent to $M(\bar{\theta})=\phi_{1}(+\infty, v) \lesssim c$.

Moreover, the properties of $\phi_{0}$ imply that the remaining admissibility inequalities hold for every $x_{0} \in \mathbb{R}$ iff

$$
\frac{1-a}{a} \sum_{k=1}^{+\infty} a^{k} \sum_{n \in \mathbb{Z}} \ell_{n<\left\lceil\frac{v k}{2}\right\rceil} \ell_{2 n, k}=\phi_{0}(0-0, v)=\phi_{0}(0, v-0) \lesssim c \leqslant \phi_{0}(0, v)=\frac{1-a}{a} \sum_{k=1}^{+\infty} a^{k} \sum_{n \in \mathbb{Z}: n \leqslant\left\lfloor\frac{v k}{2}\right\rfloor} \ell_{2 n, k}
$$

We conclude that there exists $v \in \mathbb{R}$ such that the code (8) solves the admissibility equation iff

$$
\max \left\{\phi_{1}(+\infty, v), \phi_{0}(0, v-0)\right\} \lesssim c \leqslant \phi_{0}(0, v) .
$$

The assumption that the coefficients $\ell_{n}$ are non trivial implies that $n_{\min }=\inf \left\{n \in \mathbb{Z}: \ell_{n}>0\right\}<n_{\max }$. In the remaining of the proof, we assume for convenience that $n_{\min }>-\infty$ and $n_{\max }<+\infty$. The other cases where one or both of these quantities are infinite can be treated similarly.

The function $v \mapsto \phi_{0}(0, v)$ - which is right continuous - is strictly increasing on the interval $\left[n_{\min }, n_{\max }\right]$ (this fact is shown at the end of the proof below). Hence, the velocity $v(c)$ for which the admissibility equation holds is unique when $c$ (and $a,\left\{\ell_{n}\right\}_{n \in \mathbb{Z}}$ ) is fixed.

Similarly to as before, we have $\phi_{0}\left(0, n_{\max }\right)=\frac{1-a}{a} \sum_{k=1}^{+\infty} a^{k} \sum_{n \in \mathbb{Z}} \ell_{2 n, k}=m(\bar{\theta})$. Let $v_{\min }=\inf \left\{v \geqslant n_{\min }:\right.$ $\left.\phi_{0}(0, v) \geqslant M(\bar{\theta})\right\}$. For the sake of clarity, we also assume here that $v_{\min }>-\infty$. (The case $v_{\min }=-\infty$ (which can only happen when $n_{\min }=-\infty$ ) can be equally treated without additional difficulties.) The velocity $v(c)$ is given by

$$
v(c)=\inf \left\{v \in\left[v_{\min }, n_{\max }\right]: \phi_{0}(0, v) \geqslant c\right\}
$$

and the front exists iff $\phi_{0}(0, v(c)-0) \lesssim c$. Therefore for each $c \in[M(\bar{\theta}), m(\bar{\theta})]$, there exists $v \in\left[v_{\min }, n_{\max }\right]$ such that the code (8) satisfies the admissibility equation, except if $\phi_{0}(0, v(c)-0)=c$ when this limit is reached (and if $c=M(\bar{\theta})$ when $v_{\min }=n_{\max }$ ). This occurs iff $\frac{p}{q} \in\left[v_{\min }, n_{\max }\right] \cap \mathbb{Q}$ when $v_{\min }<n_{\max }$, i.e. we have

$$
G=\bigcup_{\frac{p}{q} \in\left[v_{\min }, n_{\max }\right] \cap \mathbb{Q}} \phi_{0}\left(0, \frac{p}{q}-0\right)
$$

which is countable and nowhere dense. (If $v_{\min }=n_{\max }$, we have $G=M(\bar{\theta})$ which is obviously countable and nowhere dense.) This proves statements (i), (ii) and (iii), excepted the continuous dependence on $a$ and $\left\{\ell_{n}\right\}_{n \in \mathbb{Z}}$. However, this property can be easily proved following the same lines as in the proof of front's velocity continuity in [15]. The details are left to the reader.

It remains to prove that $v \mapsto \phi_{0}(0, v)$ is strictly increasing on $\left[n_{\min }, n_{\max }\right]$. Given $v_{1}<v_{2}$ in this interval, we obtain using the expression above of $\phi_{0}(0, v)$

$$
\phi_{0}\left(0, v_{2}\right)-\phi_{0}\left(0, v_{1}\right)=\frac{1-a}{a} \sum_{k=1}^{+\infty} a^{k} \sum_{\left\lfloor\frac{v_{1} k}{2}\right\rfloor<n \leqslant\left\lfloor\frac{v_{2} k}{2}\right\rfloor} \ell_{2 n, k} \geqslant \frac{1-a}{a} \sum_{k=1}^{+\infty} a^{2 k} \sum_{\left\lfloor v_{1} k\right\rfloor<n \leqslant\left\lfloor v_{2} k\right\rfloor} \ell_{2 n, 2 k}
$$

Moreover, an induction based on relation (3) shows that if $n_{0}, n_{1} \in \mathbb{Z}$ are such that $\ell_{n_{0}}>0$ and $\ell_{n_{1}}>0$, then we have

$$
\ell_{p n_{0}+(m-p) n_{1}, m}>0, \forall p \in\{0, \cdots, m\}, m \geqslant 1 .
$$

By definitions of $n_{\min }$ and $n_{\max }$, let the integers $n_{0}, n_{1}$ be also such that $n_{0} \leqslant v_{1}<v_{2} \leqslant n_{1}$. When $k$ is sufficiently large, there exists an integer $q$ such that

$$
\left\lceil\frac{k n_{1}-\left\lfloor v_{1} k\right\rfloor}{n_{1}-n_{0}}\right\rceil-1>q \geqslant\left\lceil\frac{k n_{1}-\left\lfloor v_{2} k\right\rfloor}{n_{1}-n_{0}}\right\rceil
$$

i.e. such that $\left\lfloor v_{1} k\right\rfloor<q n_{0}+(k-q) n_{1} \leqslant\left\lfloor v_{2} k\right\rfloor$. The conditions $n_{0} \leqslant v_{1}$ and $v_{2} \leqslant n_{1}$ guarantee that $q \in\{0, \cdots, k\}$. Applying inequality (10) with $m=2 k$ and $p=2 q$ then implies that $\ell_{2 q n_{0}+2(k-q) n_{1}, 2 k}>0$ from which it easily follows that $\phi_{0}\left(0, v_{2}\right)-\phi_{0}\left(0, v_{1}\right)>0$ as desired. 


\section{Fronts between more general periodic patterns}

The bounded orbit associated with the code (8) is actually a special case of fronts between periodic patterns that the recursion (1) may show. Indeed, the arguments in the previous section are general enough to be extended without any additional major difficulty to fronts between more general ground patterns.

Let $L \geqslant 1$ be arbitrary and let $S \subset \mathbb{Z}$ be a $L$-periodic subset (i.e. $s \in S$ iff $s+L \in S$ ) that does not intersect the set $L \mathbb{Z}$ composed of multiple integers of $L$. The ground pattern behind (resp. ahead of) the front is the stationary solution with spatial code $\underline{\theta}=\left\{\chi_{S}(s)\right\}_{s \in \mathbb{Z}}\left(\right.$ resp. $\bar{\theta}=\left\{\chi_{S \cup L \mathbb{Z}}(s)\right\}_{s \in \mathbb{Z}}$ ), where $\chi_{X}$ is the characteristic function of the set $X$. The front itself is the bounded orbit associated with the code

$$
\theta_{s}^{t}=\chi_{S}(s)+\chi_{L \mathbb{Z}}(s) H\left(s-v t+x_{0}\right), \forall(s, t) \in \mathbb{Z}^{2},
$$

for some $x_{0} \in \mathbb{R}$.

This framework includes in particular standard fronts, which can be recovered by choosing $S=\emptyset$ and $L=1$, and the fronts between $0^{\infty}$ and $(10)^{\infty}$, which are obtained for $S=\emptyset$ and $L=2$. More examples of solution profiles for the recursion (2) are given in Figure 4 (see again [14] for related computer animations).
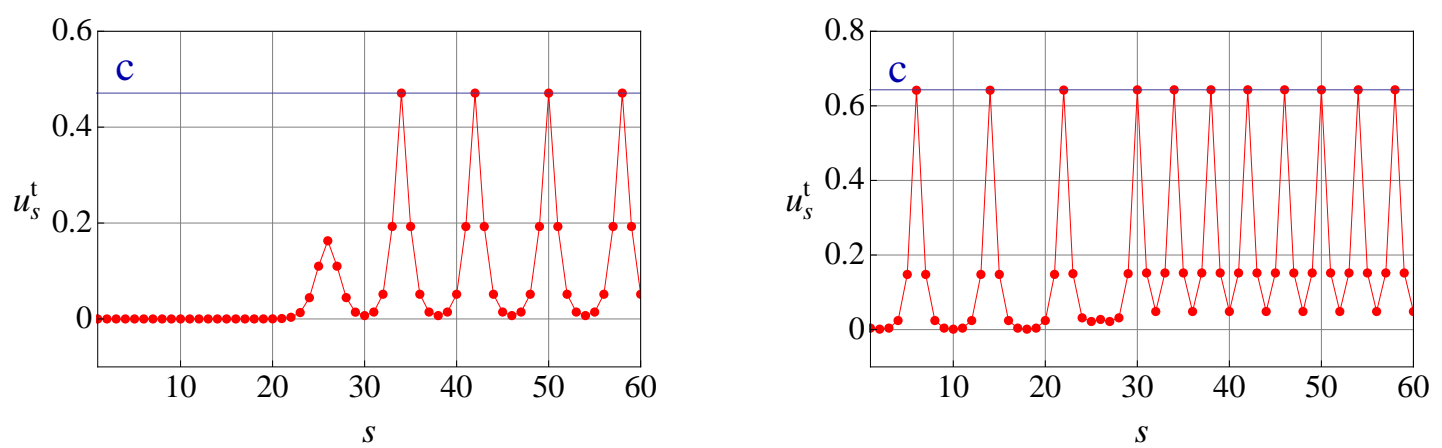

Figure 4: Snapshots of the front (11) between the ground patterns $\underline{\theta}=\left\{\chi_{S}(s)\right\}_{s \in \mathbb{Z}}$ and $\bar{\theta}=\left\{\chi_{S \cup L \mathbb{Z}}(s)\right\}_{s \in \mathbb{Z}}$ for the recursion (2). The front velocity is $v=2 / 11$ in both cases and the system parameters are $a=0.8$, $\epsilon=0.25$ and $c=0.471$ (left) $/ \epsilon=0.12$ and $c=0.643$ (right). Left picture: $S=\emptyset$ and $L=8$, i.e. front between $0^{\infty}$ and $(10000000)^{\infty}$. Right picture: $S=8 \mathbb{Z}+4$ and $L=8$, i.e. front between $(10000000)^{\infty}$ and $(1000)^{\infty}$

The existence of fronts between general ground patterns and the dependence of their velocity on parameters is given in the next statement.

Theorem 3 Given $L \geqslant 1$ and a L-periodic set $S$ not intersecting $L \mathbb{Z}$, assume that the parameter a and $\left\{\ell_{n}\right\}_{n \in \mathbb{Z}}$ are such that the inequalities $M(\underline{\theta})<m(\underline{\theta})$ and $M(\bar{\theta})<m(\bar{\theta})$ hold and the interval

$$
I_{L, S}=[M(\underline{\theta}), m(\underline{\theta})] \cap[M(\bar{\theta}), m(\bar{\theta})],
$$

is not empty (i.e. such that both stationary solutions with spatial code $\underline{\theta}$ and $\bar{\theta}$ co-exist upon appropriate choice of c). Then the following assertions hold.

(i) There exists a countable and nowhere dense set $G_{L, S}$ such that the code (11) solves the admissibility equation (5) for some velocity $v \in \mathbb{R}$ iff $c \in I_{L, S} \backslash G_{L, S}$.

(ii) For every $c \in I_{L, S} \backslash G_{L, S}$, there exists a unique $v_{L, S}(c)$ for which (11) solves (5).

(iii) The function $v_{L, S}(\cdot)$ can be extended to the whole interval $I_{L, S}$ as an increasing Devil's staircase. It also continuously depends on a and $\left\{\ell_{n}\right\}_{n \in \mathbb{Z}}$. 


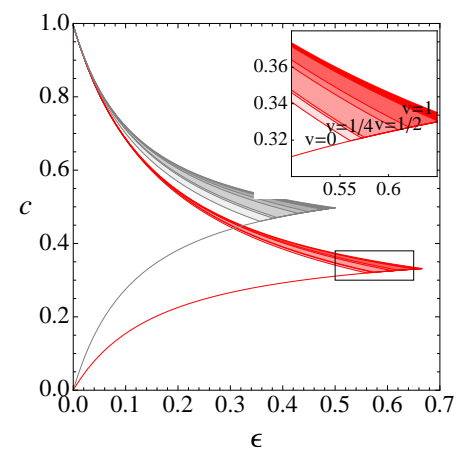

Figure 5: Existence domains in the $(\epsilon, c)$ plane for $a=0.8$ of the front between $0^{\infty}$ and $(100)^{\infty}($ i.e. $S=\emptyset$ and $L=3)$ - and gray level plot of the existence domains for the the front between $0^{\infty}$ and $(10)^{\infty}$ - for the recursion (2). Color code as in Figure 3.

Recall that we can make sure that $I_{L, S} \neq \emptyset$ for all $L, S$ by choosing the coupling operator $C$ sufficiently close to the identity, i.e. such that $l_{0}>1 / 2$. In this case, the recursion (1) has a huge variety of fronts between periodic patterns that co-exist, possibly with distinct velocities.

An illustration of the velocity domains of the front between $0^{\infty}$ and $(100)^{\infty}$ (i.e. $S=\emptyset$ and $L=3$ ) for the recursion (2), based on explicit expressions (obtained in the proof below), is given Figure 5 .

Proof of the Theorem. The proof follows the same lines as in the previous proof. When applied to the code (11), linearity of the component function $\phi$ in relation (4) implies that the corresponding bounded orbit coordinates can be written as a sum of a time-independent component and a periodic traveling wave one, i.e.

$$
u_{s L+r}^{t}=\underline{u}_{r}+\phi_{r, L}\left(s L-v t+x_{0}, v\right), \forall r \in\{0, \cdots, L-1\},(s, t) \in \mathbb{Z}^{2},
$$

where $\underline{u}_{s}=\sum_{n \in \mathbb{Z}} l_{n} \underline{\theta}_{s-n}=\sum_{n \in \mathbb{Z}} l_{n} \chi_{S}(s-n)$ are the components of the $L$-periodic background (encoded by $\underline{\theta}$ ) and

$$
\phi_{r, L}(x, v)=\frac{1-a}{a} \sum_{k=1}^{+\infty} a^{k} \sum_{n \in \mathbb{Z}} \ell_{n L+r, k} H(x-n L+v k), \forall x, v \in \mathbb{R} .
$$

Using that the code (11) rewrites under the following form

$$
\theta_{s L}^{t}=H\left(s L-v t+x_{0}\right) \text { and } \theta_{s L+r}^{t}=\chi_{S}(r), \forall r \in\{1, \cdots, L-1\},(s, t) \in \mathbb{Z}^{2},
$$

the admissibility equation becomes equivalent to

$$
\sup _{s, t: s L-v t+x_{0}<0} \underline{u}_{0}+\phi_{0, L}\left(s L-v t+x_{0}, v\right)<c \leqslant \inf _{s, t: s L-v t+x_{0} \geqslant 0} \underline{u}_{0}+\phi_{0, L}\left(s L-v t+x_{0}, v\right),
$$

and

$$
H\left(\underline{u}_{r}+\phi_{r, L}\left(s L+r-v t+x_{0}, v\right)\right)=\chi_{S}(r), \forall r \in\{1, \cdots, L-1\},(s, t) \in \mathbb{Z}^{2} .
$$

Similarly to as in the previous proof, each function $x \mapsto \phi_{r, L}(x, v)$ is right continuous and strictly increasing, and reaches its limit $\phi_{r, L}(+\infty, v)$ iff $v=n_{\max }$. Moreover, we have $\phi_{r, L}(-\infty, v)=0$ for all $v \in \mathbb{R}$. Accordingly, the last condition is equivalent to

$$
\max _{r \in\{1, \cdots, L-1\} \backslash S} \underline{u}_{r}+\phi_{r, L}(+\infty, v) \lesssim c \leqslant \min _{r \in S} \underline{u}_{r}=m(\underline{\theta})
$$

where $\max _{r \in\{1, \cdots, L-1\} \backslash S} \underline{u}_{r}+\phi_{r, L}(+\infty, v)=\max _{r \in \mathbb{Z} \backslash S} \bar{u}_{r}=M(\bar{\theta})$. Moreover, as in the previous proof, monotonicity and right continuity imply that the inequalities in the admissibility condition are equivalent to

$$
\underline{u}_{0}+\phi_{0, L}(0, v-0) \lesssim c \leqslant \underline{u}_{0}+\phi_{0, L}(0, v) .
$$

Strict monotonicity of the function $v \mapsto \phi_{0, L}(0, v)$ then implies that the front velocity is uniquely defined by

$$
v_{L, S}(c)=\min \left\{v \in\left[v_{\min }, v_{\max }\right]: \underline{u}_{0}+\phi_{0, L}(0, v) \geqslant c\right\}
$$


where

$$
v_{\min }=\inf \left\{v \geqslant n_{\min }: \underline{u}_{0}+\phi_{0, L}(0, v) \geqslant M(\bar{\theta})\right\} \text { and } v_{\max }=\sup \left\{v \leqslant n_{\max }: \underline{u}_{0}+\phi_{0, L}(0, v) \leqslant m(\underline{\theta})\right\} .
$$

(As before, the definition here assumes that both $v_{\min }$ and $v_{\max }$ are finite. Otherwise, the interval $\left[v_{\min }, v_{\max }\right]$ needs to be replaced by the appropriate one; i.e. $\left(-\infty, n_{\max }\right],\left[n_{\min },+\infty\right)$ or $(-\infty,+\infty)$ upon case.) In addition, the front with velocity $v_{L, S}(c)$ exists iff $c \gtrsim \underline{u}_{0}+\phi_{0, L}(0, v(c)-0)$. Since the limit here is attained only when the velocity is rational, it follows that

$$
G_{L, S}=\bigcup_{\frac{p}{q} \in\left[v_{\min }, v_{\max }\right] \cap \mathbb{Q}} \underline{u}_{0}+\phi_{0, L}\left(0, \frac{p}{q}-0\right)
$$

when $v_{\min }<n_{\max }$ (and $G_{L, S}=M(\bar{\theta})$ if $v_{\min }=v_{\max }=n_{\max }$ ). The rest of the proof is as before.

\section{Concluding remarks}

When $L>1$ the conditions on $a$ and $\left\{\ell_{n}\right\}_{n \in \mathbb{Z}}$ for which the interval $I_{L, S}$ in Theorem 3 is non-empty (i.e. where the corresponding ground patterns co-exist), is substantially smaller than the corresponding interval $I_{1, \emptyset}=(0,1)$ of the standard fronts, see Figures 3 and 5. Accordingly, fronts between periodic patterns may seem hardly observable in practice. However, these fronts are generically asymptotically stable and robust solutions of bistable recursions on lattices, as we argue now. As such they are likely to be seen provided that parameters and initial conditions are chosen (anywhere) in suitable open sets.

Indeed, when the front velocity is rational and the front $\left\{u_{s}^{t}\right\}_{(s, t) \in \mathbb{Z}^{2}}$ remains bounded away at positive distance from the discontinuity, i.e.

$$
\inf _{(s, t) \in \mathbb{Z}^{2}}\left|u_{s}^{t}-c\right|=\delta>0,
$$

(these properties are satisfied for every $c$ in a dense countable union of intervals in $I_{L, S} \backslash G_{L, S}$ ), it is direct to show that this solution attracts every trajectory in a $\delta$-neighborhood i.e. for an initial condition $\widetilde{u}^{0}$ such that $\left\|\tilde{u}^{0}-u^{0}\right\|<\delta$, we have

$$
\left\|\tilde{u}^{t}-u^{t}\right\|<a^{t} \delta, \forall t \geqslant 1 \text { and then } \lim _{t \rightarrow \infty}\left\|\tilde{u}^{t}-u^{t}\right\|=0 .
$$

Lyapunov asymptotic stability is proved. In addition, standard arguments based on the condition (12) also implies robustness. At first, this condition states that no front component can visit the interval $[c-\delta, c+\delta]$ around the discontinuity. Hence, the bistable nonlinearity can be modified in this interval in such a way to make $f$ be continuous (or even smooth), without affecting the front dynamics.

More generally, under condition (12), the front, which has velocity $p / q$ for some integer pair $p, q$, is actually a linearly stable space-time periodic orbit of the recursion (1), i.e. we have $u_{s-p}^{t+q}=u_{s}^{t}$. The implicit function theorem [33] implies that this space-time periodic solution can be continued for the lattice recursion

$$
\left\{u_{s}\right\}_{s \in \mathbb{Z}} \mapsto\left\{\sum_{n \in \mathbb{Z}} \ell_{n} f\left(u_{s-n}\right)\right\}_{s \in \mathbb{Z}}
$$

where $f$ is any small $C^{1}$-perturbation of $f_{a, c}$ on the set $[-\delta, c-\delta / 2] \cup[c+\delta+2,1+\delta]$. In other words, fronts between periodic patterns, with rational velocity, exist for some continuous/smooth bistable recursions on the lattice, and they are robust to small variations of the bistable nonlinearity.

Naturally, the arguments here apply to recursions in a small neighborhood of the piecewise affine mapping (1). A proper reasoning needs to be developed for more general bistable mappings on the lattice. This will be the subject of future studies.

\section{References}

[1] V.S. Afraimovich and L. Bunimovich. Density of defects and spatial entropy in extended systems. Physica D, 80:277-288, 1995. 
[2] V.S. Afraimovich and B. Fernandez. Topological properties of linearly coupled expanding map lattices. Nonlinearity, 13:973-993, 2000.

[3] V.S. Afraimovich, L.Y. Glebsky, and V.I. Nekorkin. Stability of stationary states and topological spatial chaos in multidimensional lattice dynamical systems. Random Comput. Dynam., 2:287-303, 1994.

[4] D.G. Aronson and H.F. Weinberger. Nonlinear diffusion in population genetics, combustion, and nerve pulse propagation. In Partial differential equations and related topics, volume 446 of Lec. Notes Math., pages 5-49. Springer, 1975.

[5] P.C. Bates, X. Chen, and A. Chmaj. Traveling waves of bistable dynamics on a lattice. SIAM J. Math. Anal., 35:520-546, 2003.

[6] P.C. Bates, P.C. Fife, X. Ren, and X. Wang. Traveling waves in a convolution model for phase transitions. Arch. Rat. Mech. Anal., 138:105-136, 1997.

[7] H. Berestycki, F. Hamel, and L. Roques. Analysis of the periodically fragmented environment model : Ii - biological invasions and pulsating travelling fronts. J. Math. Pures App., 84:1101-1146, 2005.

[8] J-R. Chazottes and B. Fernandez, editors. Dynamics of Coupled Map Lattices and of Related Spatially Extended Systems, volume 671 of Lect. Notes Phys. Springer-Verlag, 2005.

[9] X. Chen. Existence, uniqueness, and asymptotic stability of traveling waves in nonlocal evolution equations. Adv. Diff. Eqns, 2:125-160, 1997.

[10] S-N. Chow, J. Mallet-Paret, and E.S. Van Vleck. Pattern formation and spatial chaos in spatially discrete evolution equations. Random Comput. Dynam., 4:109-178, 1996.

[11] P. Collet and J-P. Eckmann. Instabilities and Fronts in Extended Systems. Princeton University Press, 1990.

[12] S. Coombes and C.R. Laing. Pulsating fronts in periodically modulated neural field models. Phys. Rev. E, 83:011912, 2011.

[13] P. Coullet, C. Elphick, and D. Repaux. Nature of spatial chaos. Phys. Rev. Lett., 58:431, 1987.

[14] R. Coutinho and B. Fernandez. http://www.cpt.univ-mrs.fr/ bastien/Fronts.html.

[15] R. Coutinho and B. Fernandez. Extended symbolic dynamics in bistable cml: Existence and stability of fronts. Physica D, 108:60-80, 1997.

[16] R. Coutinho and B. Fernandez. On the global orbits in a bistable cml. Chaos, 7:301-310, 1997.

[17] R. Coutinho and B. Fernandez. Fronts and interfaces in bistable extended mappings. Nonlinearity, 11:1407-1433, 1998.

[18] R. Coutinho and B. Fernandez. Fronts in extended systems of bistable maps coupled via convolutions. Nonlinearity, 17:23-47, 2004.

[19] R. Coutinho, B. Fernandez, R. Lima, and A. Meyroneinc. Discrete time piecewise affine models of genetic networks. J. Math. Bio., 52:524-570, 2006.

[20] A-D. Defontaines, Y. Pomeau, and B. Rostand. Chain of coupled bistable oscillators: a model. Physica $D, 46: 201-216,1990$.

[21] J-P. Eckmann and I. Proccacia. Onset of defect-mediated turbulence. Phys. Rev. Lett., 66:891, 1991.

[22] S. Elaydi. An introduction to difference equations. Springer, 1996.

[23] P.C. Fife and J.B. McLeod. The approach of solutions of nonlinear diffusion equations to travelling front solutions. Arch. Rat. Mech. Anal., 65:335-61, 1977.

[24] F. Hamel and L. Roques. Uniqueness and stability properties of monostable pulsating fronts. J. European Math. Soc., 13:345-390, 2011. 
[25] Y. Jin and X-Q. Zhao. Spatial dynamics of a discrete-time population model in a periodic lattice habitat. J. Dynam. Diff. Eq., 21:501-525, 2009.

[26] J.P. Keener. Propagation and its failure in coupled systems of discrete cells. SIAM J. Appl. Math., 47:556-572, 1987.

[27] V. Kocic and G. Ladas. Global behaviour of nonlinear difference equations of higher order with applications. Kluwer, 1993.

[28] R. Lui. Biological growth and spread modeled by systems of recursions. Math. Biosciences, 93:269-295, 1989.

[29] J. Mallet-Paret. The global structure of traveling waves in spatially discrete dynamical systems. $J$. Dynam. Diff. Eq., 11:49-127, 1997.

[30] H.F. Weinberger. Long-time behavior of a class of biological models. SIAM J. Math. Anal., 13:353-396, 1982.

[31] H.F. Weinberger. On spreading speeds and traveling waves for growth and migration models in a periodic habitat. J. Math. Bio., 45:511-548, 2002.

[32] J. Xin. Front propagation in heterogeneous media. SIAM review, 42:161-230, 2000.

[33] E. Zeidler. Nonlinear functional analysis and its applications: I, Fixed point Theorems. Springer, New York, 1986.

[34] B. Zinner, G. Harris, and W. Hudson. Travelling fronts for the discrete fisher's equation. J. Diff. Equ., 105:46-62, 1993. 